Irish Section Meeting, 17-19 June 2015, Nutrition at key life stages: new findings, new approaches

\title{
Frequency of electronic dietary self monitoring using a smartphone app and weight loss
}

\author{
M.C. Carter ${ }^{1}$, V.J. Burley ${ }^{1}$ and J.E. Cade ${ }^{1}$ \\ ${ }^{1}$ Nutritional Epidemiology Group, School of Food Science \& Nutrition, University of Leeds
}

Obesity is a major global public health issue due to its association with a number of serious chronic illnesses and its high economic burden to healthcare providers. Self monitoring (deliberately observing and recording behaviour) has been ascribed great importance in behavioural approaches to obesity due to its consistent associations with weight loss ${ }^{(1)}$. However, there is limited evidence about how frequently individuals need to monitor their diet for optimal weight loss. Recording diet using a smartphone application (app) allows for a more objective assessment of self monitoring given that electronic records can be time and date stamped.

A randomised pilot trial comparing 3 methods of self monitoring (smartphone app, paper diary, web-based) has been conducted ${ }^{(2)}$. Trial duration was 6 months. The smartphone app 'My Meal Mate' (MMM) features an electronic food diary and users are required to select and $\log$ food and drink items from a 23,000 item food composition database ${ }^{(3)}$. All food consumption data is automatically uploaded with a time and date stamp. Post-hoc analysis of app usage patterns was undertaken ( $\mathrm{n}=43,77 \%$ female, $100 \%$ white ethnic origin, mean age 41 (SD: 9) years, mean BMI: 34 (SD: 4) kg/m²) in order to explore the relationship between frequency of electronic dietary self monitoring and weight loss.

Table 1: Regression analysis of the relationship between frequency of use category and follow up weight (kg) (adjusted for baseline weight) after 6 months use of 'My Meal Mate' (MMM) a smartphone app for weight loss. *A complete day of dietary self monitoring was considered to be one with a biologically plausible energy intake recorded ( $\geq 500$ and $\leq 5000 \mathrm{kcal} / \geq 2093 \mathrm{kj}$ and $\leq 20934$ ).

\begin{tabular}{llll}
\hline Frequency of app usage over 6 months* & N & Weight loss $(\mathrm{kg})$ & $\mathrm{p}$ \\
\hline Low $(\leq 42$ days of dietary self monitoring) & 14 & reference & $95 \%$ CI \\
Moderate ( $\geq 43$ days to 128 days) & 14 & $-1 \cdot 8$ & $-5 \cdot 3,1 \cdot 8$ \\
High $(\geq 129$ day) & 15 & $-6 \cdot 4$ & $-10 \cdot 0,-2 \cdot 9$ \\
\hline
\end{tabular}

Regression analysis showed that those in the highest 'frequency of use' category had a $-6.4 \mathrm{~kg}(95 \% \mathrm{CI} ;-10 \cdot 0,-2 \cdot 9)$ lower follow up weight (adjusted for baseline weight) than those in the lowest 'frequency of use' category $(\mathrm{p}<0 \cdot 01)$. Results must be interpreted with caution given the small numbers in each category but this post-hoc exploratory analysis indicates that higher frequency of smartphone app use is associated with improved weight loss.

This study was funded by the National Institute for Health Research (NIHR) (ref: G0802108).

1. Burke L.E., et al. (2011) J Am Diet Assoc 111, 92-102.

2. Carter M.C., et al., (2013) J Med Internet Res 15, e32.

3. Carter M.C. et al. (2013) Nutrition Bulletin 38, 80-84. 